\title{
Securing the airway in a child with tracheal agenesis - an alternative perspective
}

\author{
Dalibor Murgas ${ }^{1}$, Martin Ciljak², Milan Dragula1, Katarina Matasova², Mirko Zibolen², Slavomir Nosal', Julian Hamzik \\ 1Department of Paediatric Surgery, Faculty Hospital, Martin, Slovakia \\ 2Department of Neonatology, Faculty Hospital, Martin, Slovakia \\ 3Department of Paediatrics, Faculty Hospital, Martin, Slovakia
}

Videosurgery and other miniinvasive techniques 2011; 6 (1): 24-26

DOI: 10.5114/wiitm.2011.20989

\begin{abstract}
Tracheal agenesis is an extremely rare, typically fatal congenital anomaly characterised by typical clinical manifestation, failed endotracheal intubation and difficult emergency management. The prognosis and possibility of surgical correction rest on early diagnosis, anatomy, birth weight, and associated anomalies. Tracheal agenesis causes considerable resuscitation difficulties immediately after birth, as tracheal intubation is impossible. Although emergency management, by either bag and mask ventilation or oesophageal intubation, provides some pulmonary gas exchange through tracheo-oesophageal connection, they are associated with overdistension of the stomach and the risk of stomach perforation. We have performed selective intubation of the distal trachea in a child with tracheal agenesis through the tracheo-oesophageal connection with the help of a fibroendoscope. A standard guide wire was passed through the working port of the flexible endoscope and under direct vision was directed through the tracheooesophageal connection into the distal trachea. At this point, the endoscope was removed, leaving the guide wire in place. The endotracheal tube size 2 Fr was passed over the guide wire into the distal trachea. The infant showed dramatic improvement and maintained good saturations on mechanical ventilation. The infant was taken for a computed tomography (CT) scan, which showed Floyd's type II tracheal agenesis, the oesophagus connecting with the distal trachea before it bifurcates. The endotracheal tube was seen ending in the carina. Transoesophageal intubation of the short distal segment of the trachea through the tracheo-oesophageal connection might offer a new perspective for short-term securing of the airway in a child with tracheal agenesis and should be considered in any child with suspected tracheal agenesis to buy the time while evaluating the exact anatomy of the anomaly and the possibility of tracheal reconstruction in selected cases.
\end{abstract}

Key words: tracheal agenesis, transoesophageal intubation, resuscitation.

\section{Introduction}

The most common causes of neonatal respiratory distress in the delivery room are birth asphyxia, prematurity and meconium aspiration. There are other less common causes such as diaphragmatic hernia, pulmonary hypoplasia, tracheo-oesophageal fistula (TOF) and choanal atresia. In all these cases classical resuscitative manoeuvres tend to improve the situation. Standard neonatal resuscitation manuals do not go into depth about what to do in cases of impossible intubation.

Tracheal agenesis (TA) is a congenital anomaly causing considerable resuscitation difficulties immediately after birth, as tracheal intubation is impossible. Although bag and mask ventilation and oeso- 
phageal intubation provide some pulmonary gas exchange through the tracheo-oesophageal connection, they are associated with stomach overdistension and risk of stomach perforation.

\section{Case report}

A male infant was born at 32 weeks' gestation by normal vaginal delivery. Routine antenatal ultrasounds throughout the pregnancy were normal. At birth, the infant was cyanosed and had irregular breathing movements. There was no cry and the Apgar scores were 6 at 1 and 7 at 5 min respectively. The infant was shifted to the neonatal intensive care unit, where repeated attempts of oro-tracheal intubation failed. Oxygen saturations were maintained above $90 \%$ through bag and mask ventilation. Visualisation of the larynx under direct vision was good and the laryngeal anatomy appeared to be normal. However, a resistance was felt in the subglottic area. Fibreoptic laryngoscopy showed normal laryngeal anatomy but the scope could not be negotiated below the cords. Keeping the diagnosis of TA with tracheo-oesophageal connection in mind, we decided to perform selective intubation of the distal trachea through the tracheo-oesophageal connection with the help of a fibroendoscope. The standard guide wire was passed through the working port of the flexible endoscope and under direct vision was directed through the tracheooesophageal connection into the distal trachea. At this point, the endoscope was removed, leaving the guide wire in place. The endotracheal tube size $2 \mathrm{Fr}$ was passed over the guide wire into the distal trachea. The infant showed dramatic improvement and maintained good saturations on mechanical ventilation. The infant was taken for a CT scan, which showed Floyd's type II tracheal agenesis, the oesophagus connecting with the distal trachea before it bifurcates. The endotracheal tube was seen ending in the carina.

Chromosomal analysis showed a normal male karyotype (46, XY). Cranial ultrasound and echocardiogram were normal. An associated anomaly was radial ray defect. After extensive consultations, in the light of uniformly poor surgical results for this type of anomaly, the decision not to attempt surgical correction was made jointly with the parents and medical teams. The infant survived for 102 hours and the parents refused our request for an autopsy.

\section{Discussion}

Tracheal agenesis is an extremely rare, typically fatal congenital anomaly characterised by typical clinical manifestation, failed endotracheal intubation and difficult emergency management. The prognosis and possibility of surgical correction rest on early diagnosis, anatomy, birth weight, and associated anomalies [1].

The incidence of TA is 1 in 50000 live births, boys being affected approximately twice as often as girls [2-5]. Since the first description by Payne in 1900 [6], approximately 150 cases of TA have been reported worldwide, as reviewed by Hirakawa et al. [7].

Tracheal agenesis is usually classified according to Floyd into types I-III [8]. Type I is characterized by agenesis of the proximal trachea with a short segment of the distal trachea arising from the anterior wall of the oesophagus before dividing into the mainstem bronchi. Type II is the commonest type and consists of agenesis of the entire trachea with the main bronchi joining in the midline. A connection between the oesophagus and the carina may or may not be present, but absence of a tracheo-oesophageal connection is a rare finding. In type III, two mainstem bronchi arise separately from the oesophagus. Relative incidences of these 3 types are 13, 65, and 22\% respectively [2]. Tracheal agenesis is associated with congenital anomalies in various organ systems in $50-94 \%$ of cases [4, 9]. The cardiovascular system, respiratory tract distal to the trachea, and gastrointestinal and genitourinary systems are most commonly affected.

On many occasions, children with TA die shortly after birth and the diagnosis is a retrospective one at postmortem examination. Diagnosis of TA should be considered in a newborn with respiratory insufficiency, absence of audible crying and failure to negotiate the endotracheal tube beyond the vocal cords in the presence of normal looking laryngeal anatomy. Ventilation with bag and mask or through an oesophageal tube is partially effective because of the tracheooesophageal connection present in most cases. Other clinical signs include failure to palpate the trachea in the suprasternal space, distension of the stomach on positive pressure ventilation, and auscultation of air entry over lung fields bilaterally as well as in the epigastrium. Diagnosis of TA is usually confirmed with helical computed tomography. Endoscopy of the upper airway is essential to distinguish extensive TA 
from short segmental TA, the latter being potentially resectable.

There are no established methods for surgical repair of type II and III at this time. Various types of homologous graft such as pericardium, oesophagus, and bladder as well as synthetic materials (silicone, elastane, Dacron) have been used to reconstruct the trachea, but with disappointing results $[10,11]$. The search for suitable materials for tracheal grafts has been unsuccessful so far. Suitable grafts will have to allow for normal growth and development, and will have to be able to clear the airways from secretions and withstand pressure changes during respiration.

Management of this uniformly lethal condition is controversial [10]. A rational approach is to maintain the infant alive until all the implications are assessed [12]. Emergency management, by either bag and mask ventilation or oesophageal intubation, can be successful at times. Although bag and mask ventilation and oesophageal intubation provide some pulmonary gas exchange through the tracheo-oesophageal connection, they are associated with overdistension of the stomach and the risk of stomach perforation.

\section{Summary}

Transoesophageal intubation of the short distal segment of the trachea through the tracheooesophageal connection might offer a new perspective for short-term securing of the airway in a child with TA and should be considered in any child with suspected TA to buy the time while evaluating the exact anatomy of the anomaly and the possibility of tracheal reconstruction in selected cases.

\section{References}

1. Fraser N, Stewart RJ, Grant J, et al. Tracheal agenesis with unique anatomy. J Pediatr Surg 2005; 40: e7-10.

2. Haben CM, Rappaport JM, Clarke KD. Tracheal agenesis. J Am Coll Surg 2002; 194: 217-22.

3. Holinger PH, Johnston KC, Parchet VN, Zimmermann AA. Congenital malformations of the trachea, bronchi and lung. Ann Otol Rhinol Laryngol 1952; 61: 1159-80.

4. Evans JA, Greenberg CR, Erdile L. Tracheal agenesis revisited: analysis of associated anomalies. Am J Med Genet 1999; 82: 415-22.

5. van Veenendaal MB, Liem KD, Marres HA. Congenital absence of the trachea. Eur J Pediatr 2000; 159: 8-13.

6. Payne WA. Congenital absence of the trachea. Brooklyn Med J 1900; 14: 568.

7. Hirakawa H, Ueno S, Yokoyama S, et al. Tracheal agenesis: a case report. Tokai J Exp Clin Med 2002; 27: 1-7.
8. Floyd J, Campbell DC Jr, Dominy DE. Agenesis of the trachea. Am Rev Respir Dis 1962; 86: 557-60.

9. Chiu T, Cuevas D, Cuevas L, Monteiro C. Tracheal agenesis. South Med J 1990; 83: 925-30.

10. Buchino JJ, Meagher DP Jr, Cox JA. Tracheal agenesis: a clinical approach. J Pediatr Surg 1982; 17: 132-7.

11. Neville WE, Bolanowski PJ, Soltanzadeh H. Prosthetic reconstruction of the trachea and carina. J Thorac Cardiovasc Surg 1976; 72: 525-38.

12. Narchi H. A failed neonatal resuscitation: Tracheal agenesis. Int Pediatrics 2000; 15: 167-9. 